\title{
Monte Carlo simulation of high-pressure phase equilibria in aqueous systems
}

\author{
J. R. Errington, K. Kiyohara, K. E. Gubbins and A. Z. Panagiotopoulos ${ }^{1}$ \\ School of Chemical Engineering, Cornell University, \\ Ithaca, NY 14853-5201, USA
}

\begin{abstract}
We have used Gibbs ensemble and histogram-reweighting Monte Carlo techniques to obtain the phase behavior of water and aqueous mixtures at high pressures. These calculations are relevant for the design of high-pressure separation processes, such as supercritical fluid extraction and supercritical water oxidation. In particular, we have obtained the phase behavior of several simple point charge models of water as well as polarizable versions of these models. None of the models studied reproduce the experimentally observed phase behavior and critical parameters of water. The binary mixture water / carbon dioxide at high pressures has also been studied. Good agreement for this mixture has been obtained without use of any adjustable parameters for the mixture interactions, thus suggesting that the predictive power of molecular-based methods is greater than that of purely phenomenological approaches, even when using imperfect pure component intermolecular potential models.
\end{abstract}

Keywords: molecular simulation, histogram methods, critical state, Gibbs ensemble, vaporliquid equilibria, intermolecular potentials, water, carbon dioxide.

\footnotetext{
${ }^{1}$ To whom correspondence should be addressed, also by e-mail: thanos@ipst.umd.edu . Current address: Institute for Physical Science and Technology and Dept. of Chemical Engineering, Univ. of Maryland, College Park, Maryland 20742.
} 


\section{Introduction}

Molecular simulation methods have emerged in recent years as a valuable tool for predictions of thermophysical properties of systems based on detailed models for the intermolecular interactions. Simulations can be used to extend the range of experimental measurements - for example, the critical properties of models for long-chain normal alkanes have been obtained from simulations [1] at conditions for which experimental measurements are impossible because of thermal decomposition. Predictions of the highpressure phase behavior of fluid mixtures is a natural area of application of simulation methods, since experimentation at high pressure conditions is difficult, literature data are often sparse, and semiempirical modeling methods often fail to describe many aspects of the phase behavior. However, application of molecular simulation methods is limited by the lack of intermolecular potential models that reproduce accurately the thermodynamic properties and phase behavior of pure components and mixtures. The present work is part of an ongoing effort at Cornell to obtain intermolecular potential models that can be used for reliable predictions of properties for technologically important fluids over broad ranges of temperatures and densities.

Water and aqueous solutions are of great importance in many fields of science and engineering. Because of this, significant efforts have been made to obtain reliable intermolecular potential models for water. The SPC [2], SPC/E [3] and TIP4P [4] potentials are popular because of their simplicity and relative accuracy for properties such as the energy and the radial distribution function of the liquid at ambient conditions. However, the potential parameters of these models were obtained by fitting to experimental values in the liquid at ambient conditions and are not necessarily appropriate for calculating properties at different temperatures and densities.

Water is known to be a highly polarizable molecule and the effective intermolecular interactions vary with density and temperature. Fixed-charge models such as SPC and TIP4P do not take this variation into account. One possibility for improving simple point 
charge models for water is to introduce polarizability. A number of polarizable water models have already been proposed (for example, [5]). However, to our knowledge, the phase behavior of polarizable water models has not been studied previously.

In the present paper, we review briefly the Temperature and Hamiltonian Scaling Gibbs ensemble Monte Carlo method from which we obtain the properties of several models over a range of temperatures in a single simulation, and histogram reweighting methods that allow the accurate calculation of properties over a range of densities and temperatures from a series of grand canonical Monte Carlo calculations. The next section presents non-polarizable and polarizable intermolecular potential models for water, and results for their coexistence and critical properties. We present calculations for the $\mathrm{CO}_{2-}$ $\mathrm{H}_{2} \mathrm{O}$ binary mixture that demonstrate the predictive power of molecular simulation methods even when the intermolecular potential parameters are not perfectly known.

\section{Simulation methodologies}

\subsection{Temperature and Hamiltonian scaling Gibbs ensemble Monte Carlo}

The Gibbs ensemble methodology [6,7] is a well-established technique for molecular-based calculations of phase equilibria in fluids and mixtures. The method is based on performing a calculation in two distinct regions, coupled in a way that ensures that the conditions of phase equilibrium are satisfied in a statistical sense. In its original formulation, a single calculation gives the coexistence densities (and compositions in the case of mixtures) for a given temperature and overall density or pressure.

Valleau [8] has introduced "thermodynamic scaling Monte Carlo" methods. The methods allow sampling of multiple points in thermodynamic parameter space (e.g. different temperatures, Hamiltonians, or densities) by introducing a weighting function to

force the simulated system to sample the range of parameter space of interest. In a previous publication [9] we have combined the Gibbs ensemble Monte Carlo method with 
the concept of temperature- and Hamiltonian-scaling, thus allowing the calculation of phase equilibria for a range of temperatures and intermolecular potential models. The combined method, "Temperature and Hamiltonian Scaling Gibbs Monte Carlo" (THSG) is used in the present paper to study the properties of non-polarizable intermolecular models for water.

\subsection{Histogram reweighting}

The method has been described previously [10] - here, we would like to summarize the technique as applied to fluid systems. A grand canonical Monte Carlo simulation is performed in a simulation cell of size $V$, under periodic boundary conditions. Particles are created and annihilated using the standard acceptance criteria [11]. The frequency of occurrence of $N$ particles in the simulation cell, $f(N)$, is

$$
f(N)=Q(N, V, T) e^{\beta \mu N} / \Xi(\mu, V, T)
$$

Given the distribution function $f(N)$ (collected in histogram form in the production period of a simulation), an estimate of the ratio of canonical partition functions for the system under study for two different values of $N$ in the range covered by the simulation can be obtained directly as

$$
\frac{Q_{1}\left(N_{1}, V, T\right)}{Q_{2}\left(N_{2}, V, T\right)}=\frac{f\left(N_{1}\right)}{f\left(N_{2}\right)} e^{\beta \mu\left(N_{1}-N_{2}\right)} .
$$

In addition, one expects that a simulation at the same temperature, but at a different value of the chemical potential, $\mu^{\prime}$, would result in a new distribution function, $f^{\prime}(N)$, with

$$
\frac{f^{\prime}(N)}{f(N)} \propto e^{\beta\left(\mu^{\prime}-\mu\right) N}
$$

Rescaling can only be performed over a limited range of chemical potentials since the original simulation run only contains statistically significant values over a finite range of particle numbers $N$. For extending the range of particle numbers over which the partition 
function ratio can be determined from equation 2, several runs will need to be performed at different values of the chemical potential that result in overlapping distribution functions $f(N)$. The discussion above was performed on the basis of reweighting for a single variable, the number of particles, $N$, for reasons of illustrative simplicity. In actual practice, two dimensional histograms for the frequency of observation of a certain number of particles, $N$, and a certain energy, E, are collected. This allows for reweighting in both temperature and chemical potential. For combining results from multiple runs, the technique of Ferrenberg and Swedsen [12] is used. The method has been used recently to obtain phase coexistence properties of polarizable Stockmayer fluids [13].

\section{Intermolecular potential models studied}

A class of intermolecular potential models for water is obtained by describing the electrostatic interactions from a distribution of point charges and dispersion and repulsion interactions using simple Lennard-Jones empirical forms. A schematic of typical models is shown in Figure 1. The center for the Lennard-Jones interactions for the oxygen atom is point $\mathrm{O}$. Positive partial charges are placed at two symmetric sites, $\mathrm{H}$, and a negative charge equal to twice each of the positive charges at a position M, which may be different than the location for the center of the oxygen-oxygen Lennard-Jones interactions. Table 1 summarizes the intermolecular potential models studied in this work. These include the SPC [2], SPC/E [3] non-polarizable models, and polarizable models with the SPC and TIP4P [4] geometries as well as the Kozack-Jordan polarizable model [5]. The polarizable models with SPC and TIP4P geometry are termed, respectively, SPC/P and TIP4P/P. These models were obtained by rescaling the positive and negative charges by a constant factor, so that the experimentally measured gas-phase dipole moment $(1.85 \mathrm{D})$ is recovered for the

isolated molecule. An isotropic polarizability of $0.00144 \mathrm{~nm}^{3}$ was added at the center of Lennard-Jones interactions for oxygen. 


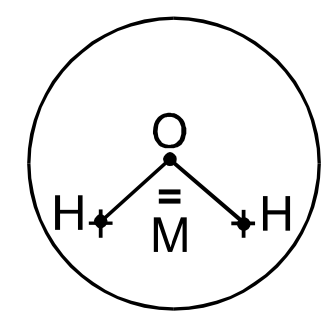

Fig. 1. Schematic geometry of water models

Table 1. Geometry and intermolecular potential parameters for water models

\begin{tabular}{|c|c|c|c|c|c|}
\hline Parameter & SPC & SPC/E & $\mathrm{SPC} / \mathrm{P}$ & $\mathrm{TIP} 4 \mathrm{P} / \mathrm{P}$ & $\mathrm{KJ}$ \\
\hline $\mathrm{r}(\mathrm{OH}), \mathrm{nm}$ & 0.1 & 0.1 & 0.1 & 0.09572 & 0.0957 \\
\hline$\angle \mathrm{HOH}$, deg. & 109.47 & 109.47 & 109.47 & 104.52 & 104.5 \\
\hline$\varepsilon_{\mathrm{OO}} / \mathrm{k}_{\mathrm{B}}, \mathrm{K}$ & 78.197 & 78.197 & 78.197 & 78.197 & 211.51 \\
\hline$\sigma_{\mathrm{OO}}, \mathrm{nm}$ & 0.3166 & 0.3166 & 0.3166 & 0.3154 & 0.317 \\
\hline Qo, e & -0.82 & -.8476 & -0.6690 & 0. & +1.2456 \\
\hline $\mathrm{Q}_{\mathrm{M}}, \mathrm{e}$ & & & & -0.8836 & -2.4912 \\
\hline $\mathrm{Q}_{\mathrm{H}}, \mathrm{e}$ & +0.41 & +0.4238 & +0.3345 & +0.4418 & +0.6228 \\
\hline $\mathrm{r}(\mathrm{OM}), \mathrm{nm}$ & & & & 0.015 & 0.0138 \\
\hline $\mathbf{m}_{0}, \mathrm{D}$ & 2.27 & 2.35 & 1.85 & 1.85 & 1.85 \\
\hline$\alpha, \mathrm{nm}^{3}$ & - & - & 0.00144 & 0.00144 & 0.00144 \\
\hline
\end{tabular}




\section{Results and discussion}

\subsection{Non-polarizable water models}

Our results for the coexistence properties of the non-polarizable water models are shown in Figure 2. Agreement between our current calculations using both the Gibbs ensemble and THSG and results from previous investigations is excellent. Some statistical noise is present in the THSG results, which are in the form of continuous curves. From analysis of results from histogram-reweighting data for SPC and SPC/E water (not shown in Figure 2) we obtain estimates of the critical properties of these models as follows. $T_{C}=593.8 \pm 1.0 \mathrm{~K}$, $\rho_{\mathrm{C}}=262.5 \pm 9.7 \mathrm{~kg} / \mathrm{m}^{3}$ (SPC) and $\mathrm{T}_{\mathrm{C}}=639.0 \pm 1.1 \mathrm{~K}, \rho_{\mathrm{C}}=262.2 \pm 8.4 \mathrm{~kg} / \mathrm{m}^{3}$ (SPC/E). These are to be compared to the experimental values of $T_{C}=647.1 \mathrm{~K}, \rho_{C}=322 \mathrm{~kg} / \mathrm{m}^{3}$. These values are slightly different than the most recent previous calculation for the SPC/E model [15] namely $\mathrm{T}_{\mathrm{C}}=630.4 \mathrm{~K}, \rho_{\mathrm{C}}=308 \mathrm{~kg} / \mathrm{m}^{3}$ (SPC/E) Both non-polarizable models underestimate the critical temperature and density, even though the SPC/E model does significantly better for the critical temperature than the SPC model. Interestingly, the model that does better for the critical temperature (SPC/E) performs less well than the SPC model for the vapor pressure. In figure 3, we show results of our calculations for the vapor pressure of the nonpolarizable water models.

\subsection{Polarizable water models}

The phase behavior of the polarizable models of Table 1 obtained from histogramreweighting GCMC calculations is shown in figure 4. As seen in figure 4, the polarizable versions of the SPC and TIP4P models underestimate significantly the critical temperature of water. From the point of view of predicting the phase behavior near the critical point, the polarizable models studied here are no better than the non-polarizable ones. The KozackJordan model overestimates the critical temperature - the model also does not do very well in predicting the structure of liquid water at ambient conditions [5]. 

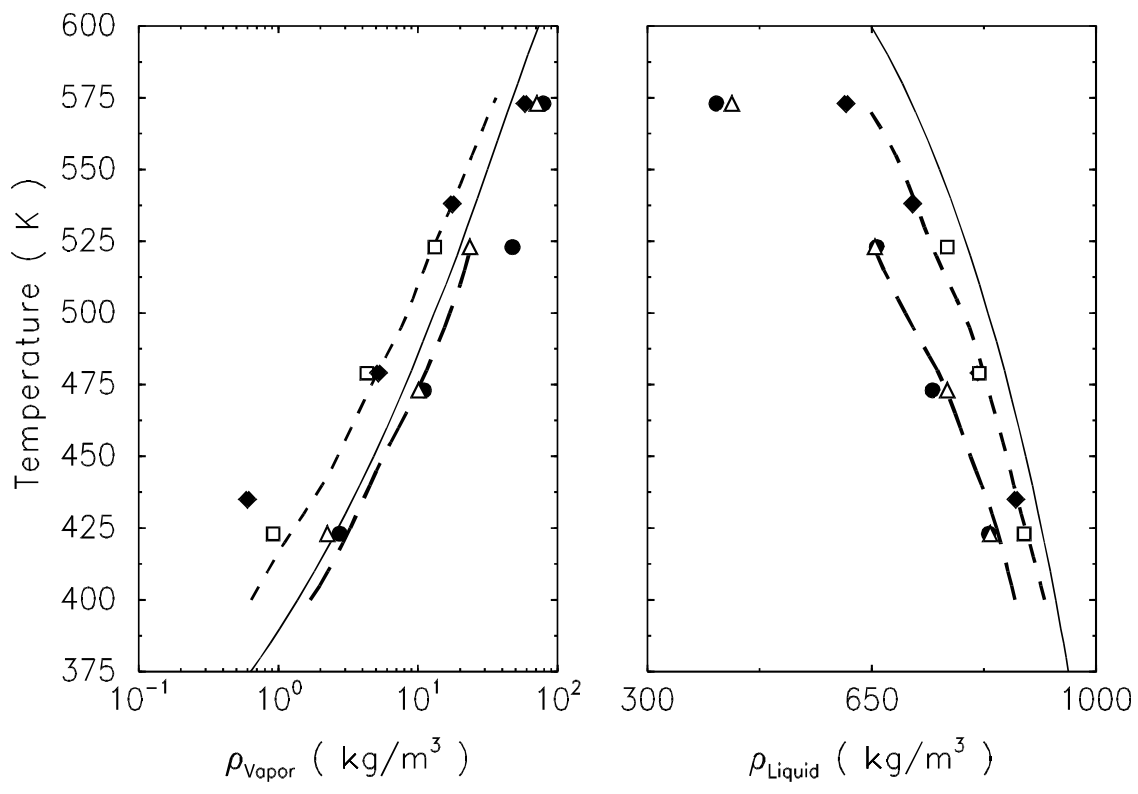

Figure 2. Saturated liquid and vapor densities for water. (-) Experimental data; For the SPC model: $(\bullet)$ de Pablo et al., [14]; $(\triangle)$ This work, Gibbs ensemble; (short dashed line) this work, THSG; For the SPC/E model: $(\diamond)$ Alejandre et al., [15]; $(\square)$ this work, Gibbs ensemble; (long dashed line) this work, THSG.

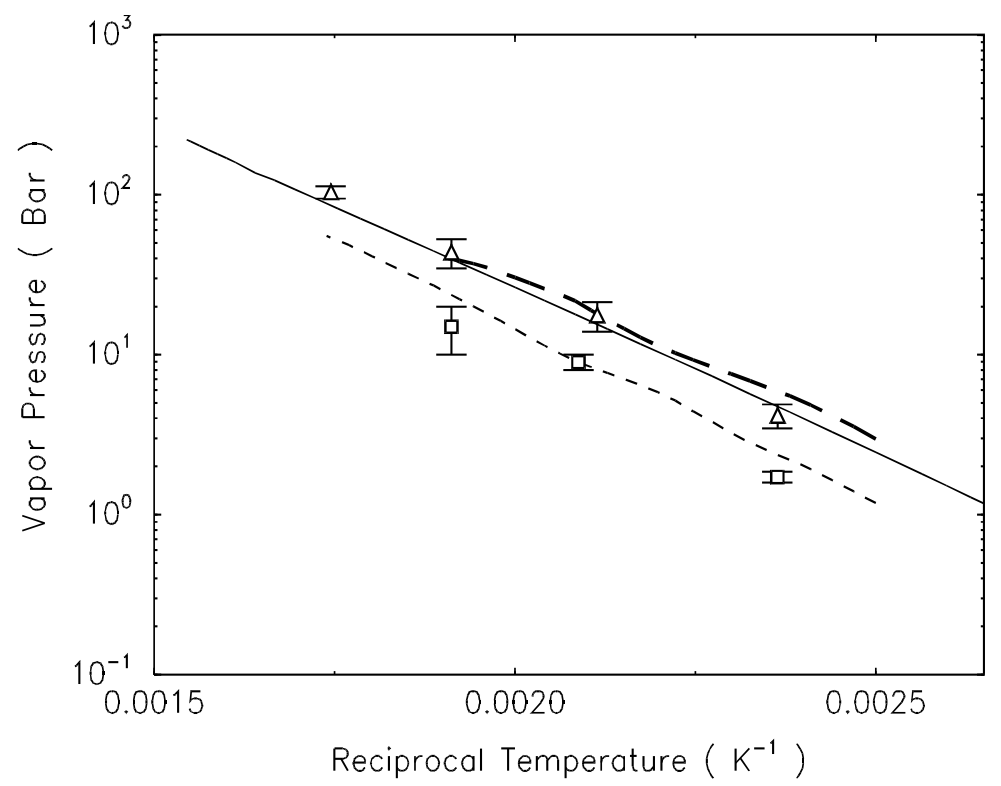

Figure 3 Vapor Pressure for water. (-) Experimental data ; For the SPC model: $(\triangle)$ This work, Gibbs ensemble; (short dashed line) this work, THSG; For the SPC/E model: ( $\square$ ) this work, Gibbs ensemble; (long dashed line) this work, THSG. 


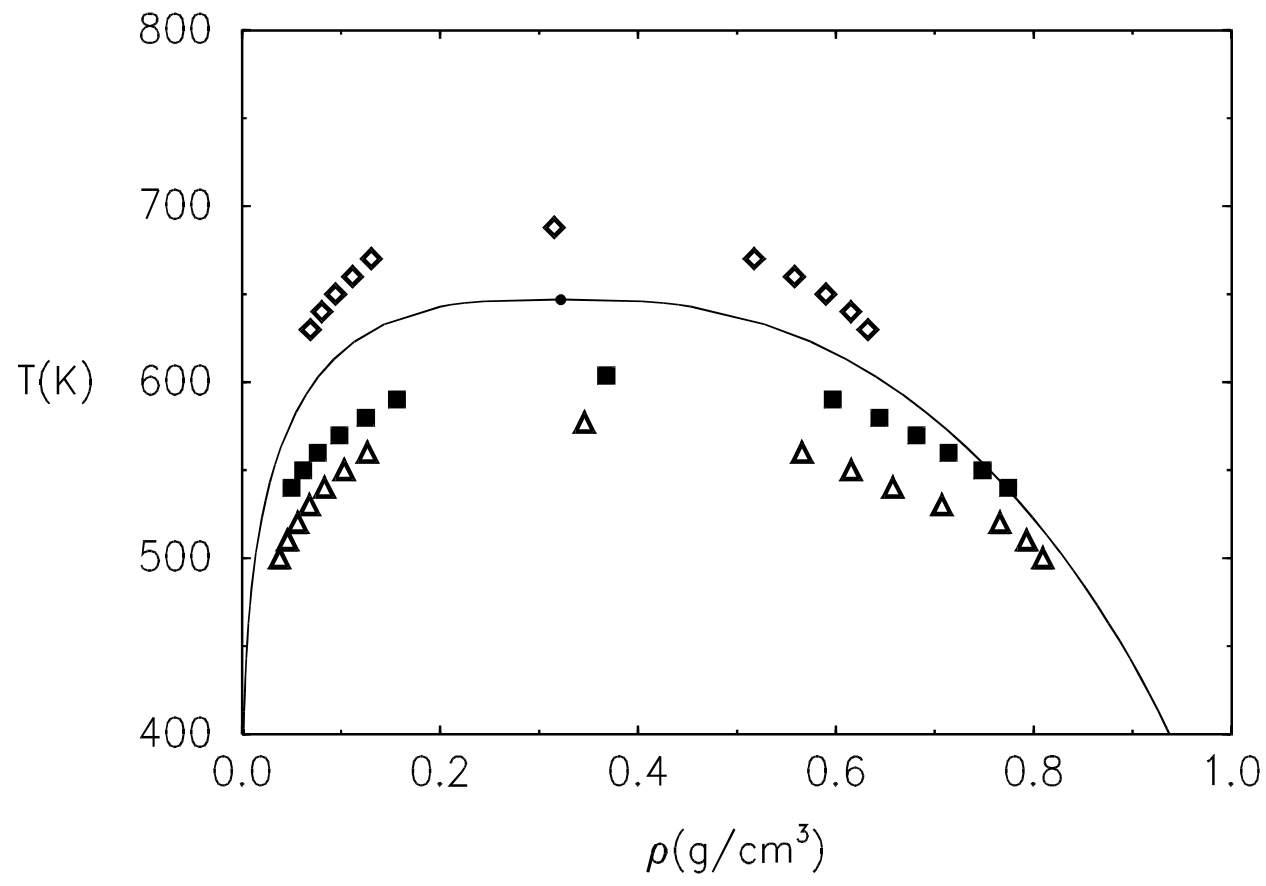

Figure 4. Phase behavior of polarizable water models from histogram reweighting GCMC simulations. (-) Experimental data ; $(\diamond) \mathrm{KJ}$ model; $(\boldsymbol{\square}) \mathrm{TIP} 4 \mathrm{P} / \mathrm{P}$ model; $(\triangle) \mathrm{SPC} / \mathrm{P}$ model. For an explanation of the models see section 3.

\subsection{Water-carbon dioxide mixtures}

Even though the models for water we have studied do not reproduce exactly the phase behavior of the pure substance, it is interesting to test how well they perform in predictions of aqueous mixture phase behavior. A mixture particularly important for high-pressure separation processes is the $\mathrm{CO}_{2}-\mathrm{H}_{2} \mathrm{O}$ binary. A good intermolecular potential model for pure $\mathrm{CO}_{2}$ is available [16]. Harismiadis et al. [17] have calculated the phase behavior for the mixture water / carbon dioxide, without using any adjustable binary parameters for the cross potential parameters. They used the SPC model for $\mathrm{H}_{2} \mathrm{O}$ and the Harris and Yung [16] model for $\mathrm{CO}_{2}$. Unlike-pair potential parameters for the Lennard-Jones interactions were calculated with the Lorenz-Berthelot rules. The coulombic interactions do not require any unlike potential parameters. Typical results are shown in Figure 5. Simulation results 
agree with the experimental solubility data within the statistical uncertainties. For other temperatures [17] the solubility of water in the $\mathrm{CO}_{2}$-rich phase at higher pressure shows deviations between experimental and calculated results. The overall agreement is quite remarkable, given that no adjustment of the intermolecular potential parameters has been performed to match experimental data. By comparison, a phenomenological equation of state with one adjustable parameter fitted to the vapor composition is unable to reproduce the data for the solubility of the two phases simultaneously. The ability to make reasonable predictions for the mixture phase behavior without adjustable parameters fitted to experimental data is expected to be one of the main benefits of molecular simulation methods.
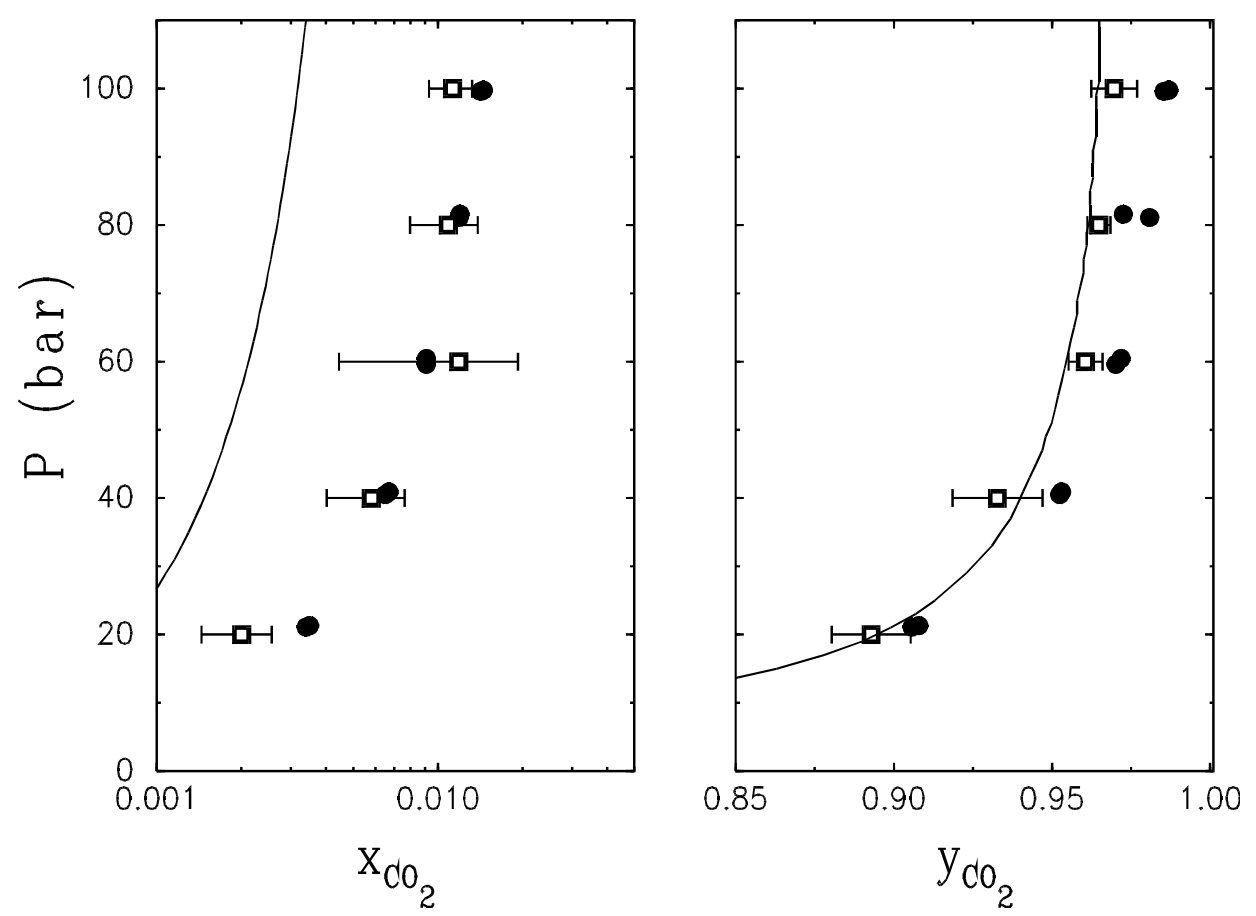

Figure 5. Phase behavior of the $\mathrm{CO}_{2}-\mathrm{H}_{2} \mathrm{O}$ system. (•) Experimental data [18,19,20]; ( $\square$ ) Gibbs ensemble calculations using the SPC model for $\mathrm{H}_{2} \mathrm{O}$ and the Harris and Yung model for $\mathrm{CO}_{2}$ [17]; (-) Prediction of the Peng-Robinson equation of state with one binary adjustable parameter. 


\section{Conclusions}

We have calculated the phase behavior near the critical region for several polarizable and non-polarizable models for water. The best simple non-polarizable model in terms of critical temperature is the SPC/E model, but the SPC model predicts the vapor pressure in better agreement to experiment over a broad temperature range. The polarizable models studied here are similar to the SPC and TIP4P non-polarizable models in geometry, but also fail to capture the phase behavior of water near the critical point.

Even with imperfect pure component models, encouraging results have been obtained for the phase behavior of binary mixtures of water and carbon dioxide, without use of adjustable parameters fitted to mixture experimental data.

\section{Acknowledgements}

Financial support for this work has been provided by the National Science Foundation and the Department of Energy (Office of Basic Energy Sciences). Supercomputer time has been provided by the Cornell Theory Center.

\section{References}

[1] Siepmann, J.I.; Karaborni, S.; Smit, B., Nature, 365 (1993) 330-2.

[2] Berendsen, H.J.C., J.P.M. Postma, W.F. van Gusteren and J. Hermans, in Intermolecular Forces, Pullmann, B. (ed.); Reidel: Dodrecht, 1981, p. 331.

[3] Berendsen, H.J.C., J.R. Grigera and T.P. Straatsma, J. Phys. Chem., 91 (1987) 626971.

[4] Jorgensen, W.L.; Chandrasekhar, J.; Madura, J.D., J. Chem. Phys., 79 (1983) 926-35.

[5] Kozack, R.E.; Jordan, P.C., J. Chem. Phys., 96 (1992) 3120-30. 
[6] Panagiotopoulos, A.Z., Molec. Phys., 61 (1987) 813-826.

[7] Panagiotopoulos, A.Z.; Quirke, N.; Stapleton, M.; Tildesley, Molec. Phys., 63 (1988) $527-545$.

[8] Valleau, J.P., J. Chem. Phys., 99 (1993) 4718-4728.

[9] Kiyohara, K., T. Spyriouni, K. E. Gubbins and A. Z. Panagiotopoulos, Molec. Phys., 89, (1996), 965-74.

[10] Ferrenberg, A.M.; Swendsen, R.H., Phys. Rev. Lett., 61 (1988) 2635-8.

[11] Frenkel, D. and B. Smit, “Understanding Molecular Simulation,” Academic Press, London, 1996.

[12] Ferrenberg, A.M. and R.H. Swendsen, Phys. Rev. Lett., 63 (1989) 1195-98.

[13] Kiyohara, K., K. E. Gubbins and A. Z. Panagiotopoulos, J. Chem. Phys., 106, (1997), 3338-47.

[14] de Pablo, J.J., J.M. Prausnitz, H.J. Strauch and P.T. Cummings, J. Chem. Phys., 93 (1990) 7355-9.

[15] Alejandre, J.; Tildesley, D.J.; Chapela, G.A., J. Chem. Phys., 102 (1995) 4574-83.

[16] Harris, J. G.; Yung, K. H., J. Phys. Chem., 99 (1995) 12021-24.

[17] Harismiadis, V. I.; Vorholz, J.; Panagiotopoulos, A.Z., Unpublished data available on the web, in http://argo.cheme.cornell.edu/gibbs/results/co2-h2o/co2-h2o.html .

[18] Wiebe, R.; Gaddy, V., J. Am. Chem. Soc., 61 (1939) 315-8.

[19] Coan, C.R.; King, A.D.,Jr., J. Am. Chem. Soc., 93 (1971) 1857-62.

[20] Tödheide, T.; Franck, E., Z. Phys. Chem. Neue Folge, 37 (1963) 387-401. 